approximates to what may be called the Rayleigh value, namely, $\mu=\nu \sigma$, which is much larger. The experimental values for the electrical resistances of liquid alkali and noble metals verify the former value, and those for liquid bismuth, lead and tin the latter, with reasonable values for $\sigma$.

The exceptional electric behaviour of mercury is due to the following circumstance. Its prominent diffraction halo, which is very sharp, is much closer to the centre than in other liquids; the spherical zone in phase space the boundary of which corresponds to this maximum can accommodate only 0.9 electrons per atom, whereas in the other liquid metals studied this number varies from $1 \cdot 2$ to 1.7 per atom.

K. S. Krishnan.

University of Allahabad,

A. B. Bhatra.

Allahabad.

June 20.

1 Proc. Nat. Acad. Sci. India, in the press. We owe to Frenkel the idea of calculating the coefficient of electronic scattering in a metal in terms of the thermal fluctuations in density.

${ }^{2}$ For collected data, see Gingrich, N. S., Rev. Mod. Phys., 15, 90 (1944).

${ }^{3}$ Ramsauer, C., and Kollath, R., Ann. Phys., 12, 529 (1932).

\section{Stability of the Earth's Atmospheric Oxygen}

Among the many ways in which it is scientifically prophesied that the world, as we know it, must come to an end, there is a double 'threat' to the oxygen of the air.

It has been argued (by Goldschmidt originally), and generally accepted, that this oxygen is already much, perhaps a half part, absorbed by iron and ferrous minerals, and must all disappear in the course of time. But those who suggest this neglect the somewhat obvious circumstance that the same gravitational differentiation which separated oxygen from the iron core originally is still in operation, although now as a very slow 'geological' effect; and that, correspondingly, ferric oxide must be dissociated at a certain depth (c. 850 miles) provided that oxygen can escape more easily than iron through the molten basalt (as is certain).

Thus, the equivalent volumes of $\mathrm{Fe}_{2} \mathrm{O}_{3}$ and $\mathrm{Fe}_{2}$ are 30.5 and $14 \cdot 3$ c.e.; the difference being $16 \cdot 2$ c.c., or at the pressure considered more like $14 \cdot 6$ c.c.; and the heat of formation of $\mathrm{Fe}_{2} \mathrm{O}_{3}$ (known at $400^{\circ} \mathrm{C}$. and not likely to be greatly different, even at $4,000^{\circ} \mathrm{C}$., from the specific heat trends, but possibly some 20-60 per cent less) is 196,000 cal. $\left(=8 \cdot 23 \times 10^{12}\right.$ ergs $)$ : from this the dissociation pressure is $8.23 \times 10^{12} \div 14 \cdot 6$ dynes $\mathrm{cm} .^{-2}$. Now, down to the depth considered, the ehanges in gravity (some 10 per cent) and magma density are not great and tend to cancel one another ; so that, for a surface basalt density of $3 \cdot 3$, the ratio

$$
8 \cdot 23 \times 10^{12}
$$
$\frac{8 \cdot 23 \times 10^{12}}{14.6 \times 3.3 \times 981} \rightarrow 1,372 \mathrm{~km}$., or 857 miles, gives a satisfactory estimate. It is very good confirmation of this that a heavier silicate-iron layer has been much supposed to begin at a depth of $1,350 \mathrm{~km}$.

The ruddy aspect of Mars has been cited as evidence of advanced formation of ferric oxide; but appropriate calculation shows that the pressure even at the centre of Mars is considerably less than that required for the dissociation-so that the cases are not analogous.

The other 'bogey' in this matter, though a slender one in its rate of action, is accredited equally or more definitely. It is argued that oxygen escapes kinetically from the 'top' of the atmosphere, at a rate, according to Maxwell's distribution law, of some 10 -hundreds $\mathrm{gm}$. sec.-1. This is no doubt the case; but what seems to be neglected is that much more oxygen is indrawn gravitationally. Even granting the latter at present, the 'escapists' would argue that it must escape somehow some time into 'infinite space'.

Now infinite space without a corresponding extension of matter is an archaic notion no longer accredited philosophically, or substantiated by observation (so far as it goes); and the gravitational oxygen intake is approximately $\pi\left(\frac{\gamma m}{v^{2}}\right)^{2} \cdot v \cdot \mathrm{PO}_{2}$ (assuming the capture radius $\frac{\gamma m}{v^{2}}$ to be virtually operative although it is only $1 / 15$ of the earth's radius, and that the surrounding oxygen density $\mathrm{PO}_{2}$ is $10^{-25}$ ), which amounts to about $1.8 \times 10^{-3}$ gm. sec.-1_a quantity unquestionably greater than the kinetic loss. (In the case of the moon, or of Mercury, the kinetic loss is the greater and the earth loses hydrogen to the point observed.)

Lest it should now be argued that too much oxygen in the air would be almost or quite as bad as none, it can be shown that all the oxygen supposed to be in space $\left(\mathrm{PO}_{2}\right.$ near the sun is about $\left.10^{-25}\right)$ can never increase the atmospheric content by more than about 23 per cent. For the solar space cell contains about $1.13 \times 10^{31} \mathrm{gm}$. of oxygen; and at a solar velocity of $20 \mathrm{~km}$. sec.-1 relative to its surroundings (it is probably less owing to similar movement of gas), the sun takes in gases $4 \times 10^{10}$ times as fast as the earth, thus leaving only $2.8 \times 10^{20} \mathrm{gm}$. of the oxygen total to accrue to the earth, which at present has $1.3 \times 10^{21} \mathrm{gm}$. (For a solar velocity relative to the gas of $10 \mathrm{~km}$. sec.-1 the possible increase would be only 3 per cent.) A similar relation will hold for any other gas, such as nitrogen or argon. Also the earth's iron core may act in a 'buffering' fashion, absorbing most of any excess over the present amount of oxygen.

The theological importance of the question of the future habitability of the earth was the origin of the studies here described-which evidently dispose of all reason for suggesting that the atmosphere is not permanent.

\author{
Hill Croft, (Allonby), \\ Mawbray Road, Maryport, \\ Cumberland. \\ July 6.
}

\section{Thermal Decomposition of $\mathrm{HgCl}_{2}$ Vapour}

IN a recent communication, Gaydon ${ }^{x}$ has shown that an absorption spectrum obtained from mercury chloride $\left(\mathrm{HgCl}_{2}\right)$ in a heated steel tube by $\mathrm{RaO}^{2}$, and attributed by the latter to diatomic $\mathrm{HgCl}$ (not to be confused with calomel $\left(\mathrm{Hg}_{2} \mathrm{Cl}_{2}\right)$ which does not exist as a gas), is really due to $\mathrm{CuCl}$ (present as an impurity). It is the purpose of this communication to show by thermodynamic calculation that there is no chance at all of obtaining the absorption spectrum of $\mathrm{HgCl}$ from thermally decomposed mercuric chloride vapour. The same is true for $\mathrm{HgBr}$ and HgL This may be surprising, because numerous diatomic radicals are known; for example, $\mathrm{OH}$, 